\title{
ANALYZING THE SPECTRAL (A)SYMMETRY OF THE MASSLESS DIRAC OPERATOR ON THE 3-TORUS
}

\author{
ELVIS BARAKOVIC AND VEDAD PASIC
}

\begin{abstract}
We analyze the spectrum of the massless Dirac operator on the 3-torus $\mathbb{T}^{3}$. It is known that it is possible to calculate this spectrum explicitly, that it is symmetric about zero and that each eigenvalue has even multiplicity. However, for a general oriented closed Riemannian 3-manifold $(M, g)$ there is no reason for the spectrum of the massless Dirac operator to be symmetric. Using perturbation theory, we derive the asymptotic formulae for its eigenvalues and prove that by the perturbation of the Euclidean metric on the 3-torus, it is possible to obtain spectral asymmetry of the massless Dirac operator in the axisymmetric case.
\end{abstract}

Mathematics subject classification (2010): 35Q41, 35P15, 58J50, 53C25, 65M60.

Keywords and phrases: Massless Dirac operator, asymmetry, spectrum, manifold, 3-torus, Galerkin method, perturbation theory.

\section{REFERENCES}

[1] M. F. Atiyah, V. K. Patodi And I. M. Singer, Spectral asymmetry and Riemannian geometry, Bull. London Math. Soc. 5 (1973), 229-234.

[2] M. F. ATiYah, V. K. Patodi And I. M. Singer, Spectral asymmetry and Riemannian geometry I, Math. Proc. Cambridge 77, 1 (1975), 43-69.

[3] M. F. Atiyah, V. K. Patodi And I. M. Singer, Spectral asymmetry and Riemannian geometry II, Math. Proc. Cambridge 78, 3 (1975), 405-432.

[4] M. F. ATIYAh, V. K. PATOdi AND I. M. Singer, Spectral asymmetry and Riemannian geometry III, Math. Proc. Cambridge 79, 1 (1976), 71-99.

[5] Christian B ÄR, The Dirac operator on space forms of positive curvature, J. Math. Soc. Jpn. 48, 1 (1996), 69-83.

[6] Elvis Barakovic ANd Vedad Pasic, Physical interpretation of pp-waves with axial torsion, in Massimo Bianchi And Remo RufFini, editors, The Fourteenth Marcel Grossmann Meeting, pages 1346-1350, World Scientific, 2017.

[7] Jean-Pierre Bourguignon and Paul Gauduchon, Spineurs, opérateurs de Dirac et variations de métriques, Commun. Math. Phys. 144, 3 (1992), 581-599.

[8] Olga Chervova, Robert J. Downes and Dmitri Vassiliev, The spectral function of a first order elliptic system, J. Spectr. Theory 3, 3 (2013), 317-360.

[9] Robert J. Downes, Michael LeVITIN AND DMitri VASSILIEv, Spectral asymmetry of the massless Dirac operator on a 3 -torus, J. Math. Phys. 54, 11 (2013), 111503, 18 pp.

[10] Robert J. Downes ANd DMitri VASSILIEv, Spectral theoretic characterization of the massless Dirac action, Mathematika 62, 3 (2016), 2041-7942.

[11] Yan-Long Fang, Michael Levitin and Dmitri Vassiliev, Spectral analysis of the Dirac operator on a 3-sphere, Oper. matrices 12, 2 (2018), 501-527.

[12] Friedrich, Thomas, Zur Abhängigkeit des Dirac-Operators von der Spin-Struktur, Colloq. Math. 48, 1 (1984), 57-62.

[13] Christian Grossmann, Hans-Görg Roos and Martin Stynes, Numerical treatment of partial differential equations, Springer-Verlag Berlin Heidelberg, 2007.

[14] Robion C. Kirby, The topology of 4-manifolds, Springer-Verlag Berlin Heidelberg, 1989. 
[15] H. Blaine Lawson and Marie-Louise Michelsohn, Spin geometry, volume 38, Princeton University Press, 1989.

[16] BRIAN J. MCCARTIN, Pseudoinverse formulation of Rayleigh-Schrödinger perturbation theory for the symmetric matrix eigenvalue problem, J. Appl. Math. 2003, 9, (2003), 459-485.

[17] VeDAD PASIC, New vacuum solutions for quadratic metric-affine gravity, University of Bath, Bath, 2009.

[18] Vedad PASIC, New vacuum solutions for quadratic metric-affine gravity - a metric affine model for the massless neutrino?, Math. Balkanica (N.S.) 24, 3-4 (2010), 329-340.

[19] VedAd PASIC AND Elvis BARAKOVIC, PP-waves with torsion: a metric-affine model for the massless neutrino, Gen. Relat. Gravit. 46, 10 (2014), Art. 1787, 27 pp.

[20] Vedad Pasic And Elvis Barakovic, Torsion Wave Solutions in Yang-Mielke Theory of Gravity, Adv. High Energy Phys. 2015, 239076 (2015), 7 pages.

[21] Vedad PASIC AND Elvis BARAKovic, Axial torsion waves in metric-affine gravity, in Massimo Bianchi AND Remo RufFInI, editors, The Fourteenth Marcel Grossmann Meeting, pages 11731178, World Scientific, 2017.

[22] Vedad Pasic, Elvis Barakovic and Nermin Okicic, A new representation of the field equations of quadratic metric-affine gravity, Adv. Math., Sci. J. 3, 1 (2014), 33-46.

[23] Vedad Pasic And DMitri Vassiliev, pp-waves with torsion and metric-affine gravity, Classical Quant. Grav. 22, 19 (2005), 3961-3975.

[24] Franz Rellich, Perturbation theory of eigenvalue problems, Gordon and Breach Science Publishers, New York, London, Paris, 1969.

[25] EdUARD StIEFEL, Richtungsfelder und Fernparallelismus in n-dimensionalen Mannigfaltigkeiten, Comment. Math. Helv. 8, 1, (1935), 305-353.

[26] A. Trautman,, The Dirac operator on hypersurfaces, Acta Phys. Polon. B 26, 7 (1995), 1283-1310. 